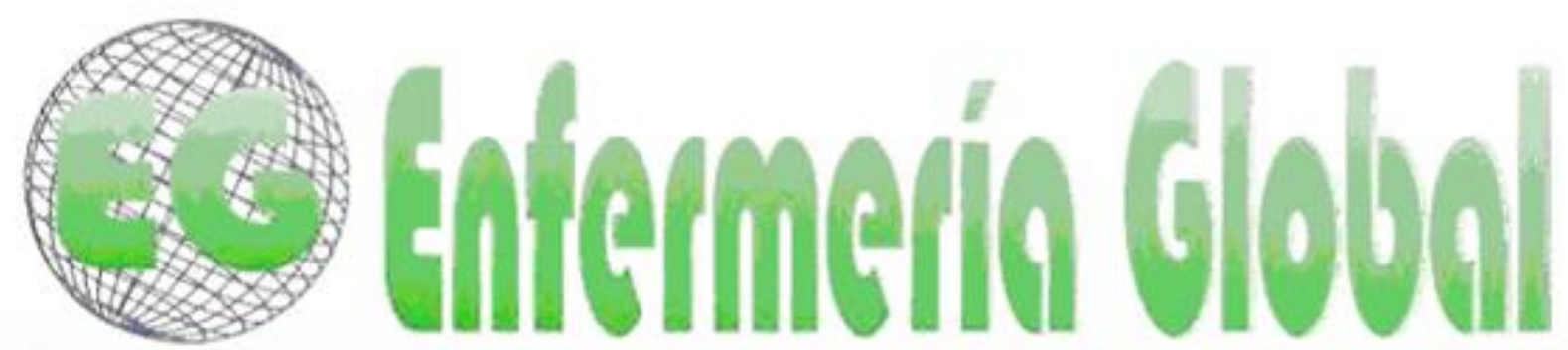

\title{
Implicaciones del hostigamiento psicológico (mobbing) sobre los testigos: Un estudio observacional en enfermería
}

Implications of Psychological Harassment on Witnesses: An Observational Study in Nursing Staff

\section{${ }^{*}$ Cardoso, Meiremar, ${ }^{* *}$ Fornés-Vives, Joana ${ }^{* *}$ Gili, Margalida}

*RN, PhD. Hospital Universitario Son Espases, Palma de Mallorca. E-mail: meiremar.cardoso@gmail.com **MSSc, PhD. Departamento de Psicología de la Universidad de las Islas Baleares. Palma de Mallorca, España.

Palabras clave: Enfermería; hostigamiento psicológico; testigos

Keywords: Nursing; psychological harassment (mobbing or bullying); witnesses.

\section{RESUMEN}

El hostigamiento psicológico en el trabajo (mobbing) es un problema de considerable magnitud para el personal de enfermería a nivel mundial. Aunque el maltrato psicológico laboral esté basado en comportamientos subjetivos, el elevado número de personas que lo testifica plantea, como mínimo, una mayor reflexión sobre este tema.

El objetivo de este estudio es analizar la problemática del mobbing desde el punto de vista exclusivo de los testigos y conocer las repercusiones psicológicas del fenómeno sobre los mismos.

Se realizó un estudio descriptivo transversal, usando cuestionarios autoadministrados. La muestra está compuesta de 204 profesionales de enfermería, de los cuales el $28.4 \%$ reportaron haber testificado conductas hostiles hacia sus compañeros de trabajo. Las conductas más observadas fueron el desprestigio profesional, la humillación, el rechazo profesional, el ninguneo y el aislamiento profesional.

Los resultados demostraron que los testigos de hostigamiento psicológico, aunque no se percibían víctimas de ello, presentaban significativamente más síntomas de estrés que los no testigos, así como un mayor deseo de abandonar la profesión y una mayor percepción de que la enfermería es una profesión poco valorada. Los resultados indican que el mobbing en enfermería es un comportamiento objetivable y que su impacto repercute negativamente en los observadores del mismo, convirtiéndolos en víctimas secundarias del fenómeno.

\section{ABSTRACT}

Psychological harassment at work (mobbing) is considered a worldwide problem of great magnitude for nursing staff. Although hostility is described as a subjective behavior, the large number of witnesses 
suggests a reflection on the phenomenon. The aim of this study is to analyze the problem of psychological harassment from the point of view of the witnesses, and to find out the psychological impact of this phenomenon on them. We performed a cross-sectional study using self-administered questionnaires. The sample consisted of 204 nurses who were non-victims of mobbing. $28.4 \%(n=58)$ of them reported to have observed hostile behavior toward coworkers. The most observed behaviors were professional discrediting, professional humiliation, rejection, scorn, and professional isolation. It was found that the witnesses of mobbing had significantly more symptoms of stress than nonwitnesses, a greater desire to leave the profession, and a greater perception that nursing is an undervalued profession. The results indicate that mobbing in nursing is an objective behavior and its impact reaches observers thereof, who may be secondary victims of the phenomenon. The results indicate that mobbing in nursing is an objective behavior and its impact reaches observers thereof, which could be considered secondary victims of the phenomenon

\section{INTRODUCCIÓN}

El hostigamiento psicológico en el trabajo, también conocido como mobbing o bullying, está considerado actualmente como una de las principales fuentes de estrés laboral $^{(1)}$, a nivel mundial. Caracterizado básicamente por comportamientos hostiles, escalonados y prolongados en el tiempo, perpetrados por una o más personas contra otra, el mobbing lleva a un aislamiento progresivo de la víctima, donde ésta se ve incapaz de defenderse. La sostenibilidad y la progresión de tales comportamientos repercuten negativamente sobre el bienestar y la salud mental de los trabajadores ${ }^{(2)}$, haciéndolos especialmente vulnerables a la agresión. Teóricamente, el hostigamiento psicológico está condicionado a la percepción de la víctima, por lo que podría contextualizarse dentro de la teoría transaccional del estrés-afrontamiento ${ }^{(3)}$, donde la evaluación subjetiva que hace la persona del evento puede determinar su reacción y su conducta.

El hostigamiento psicológico en enfermería ha sido identificado como un problema de singular magnitud ${ }^{(4)}$. Su impacto no solo afecta a la dinámica del profesional, servicio o usuarios ${ }^{(5,6)}$, sino también a los costes administrativos ${ }^{(7)}$, una vez que está asociado con la insatisfacción laboral ${ }^{(8)}$, cambios de servicio ${ }^{(9)}$, elevadas tasas de absentismo y deseo de abandonar la profesión ${ }^{(10)}$. Dichas consecuencias parecen haber influenciado, en cierta medida, las publicaciones científicas acerca de este tema, puesto que la mayoría de los estudios centran su atención sobre todo en las víctimas del maltrato laboral, y/o sobre su influencia en el entorno laboral. En este sentido, son escasos los trabajos que analizan el impacto que tiene el hostigamiento psicológico sobre los testigos ${ }^{(11)}$, lo que dificulta un análisis más detallado sobre cómo los observadores se ven influenciados por el maltrato psicológico del otro. Además, la aportación de informaciones por parte de estos observadores, siempre y cuando ellos no se perciban víctimas directas de la agresión ${ }^{(12)}$, podría suponer un avance en lo que se refiere a un acercamiento más objetivo de la hostilidad laboral. El relato de los testigos podría ofrecer cierta imparcialidad a la hora de cuantificar y categorizar las conductas hostiles en el lugar de trabajo, puesto que ellos solo se perciben cómo espectadores de dichas conductas.

Aunque podría resultar complicado asegurar la neutralidad de los testigos en cuanto a su condición de no víctima, su presencia no debería pasar inadvertida ${ }^{(13,14)}$ ya que su porcentaje es elevado. Así, las evidencias lo sitúan entre el $17,3 \%{ }^{(13)}$ y más del $80 \%^{(8,15)}$. Otros trabajos resaltan que supervisoras de enfermería ya habían sido testigos de mobbing antes de ocupar el cargo ${ }^{(16)}$, o que incluso estudiantes de enfermería y enfermeras recién graduadas eran víctimas u observadoras de maltrato psicológico ${ }^{(14)}$. Por consiguiente, la literatura científica sugiere que los comportamientos agresivos en enfermería son a menudo objetivables ya que son 
perpetrados delante de otros, lo que desencadena sentimientos de degradación y debilidad $^{(6)}$, reforzando la teoría de que éste es un comportamiento socializado ${ }^{(17)}$.

Por otra parte, hay datos de que los testigos de hostigamiento psicológico en el trabajo se perciben indirectamente afectados e intimidados por los comportamientos observados $^{(1,18)}$. En este sentido, estudio previos demuestran que los testigos padecen más problemas físicos y mentales que los no testigos, así como elevados niveles de estrés ${ }^{(2,8)}$, menor satisfacción y productividad laboral, lo que repercute negativamente sobre el desempeño de su tarea ${ }^{(5)}$ y consecuentemente en la atención prestada a los usuarios ${ }^{(18)}$.

\section{OBJETIVO DEL ESTUDIO}

El objetivo general de este estudio es analizar la problemática del mobbing desde el punto de vista de los testigos y conocer las repercusiones físicas y/o psicológicas del fenómeno sobre los mismos. Los objetivos específicos son los siguientes:

1. Determinar el porcentaje y el perfil de los testigos de hostigamiento psicológico en personal de enfermería de un hospital público de la isla de Mallorca (España).

2. Identificar los tipos de comportamientos hostiles más observados por los testigos.

3. Analizar el impacto psicológico del hostigamiento en el trabajo en aquellos que lo observan.

\section{MATERIAL Y MÉTODO}

Se ha llevado a cabo un estudio descriptivo de corte transversal. La muestra está constituida por personal de enfermería de un hospital público de la isla de Mallorca. Todos los encuestados fueron seleccionados de forma aleatoria. Como criterio de inclusión, se exigió que todos los participantes tuvieran una antigüedad mínima en la unidad de 4 semanas, con el fin de asegurar su exposición a diferentes estresores laborales, como por ejemplo, el hostigamiento psicológico ${ }^{(19)}$. Se excluyeron de la muestra todas aquellas personas que manifestaron percibirse víctimas de hostigamiento. El total anonimato de los datos ha sido garantizado a todos los participantes.

\section{Participantes}

La muestra inicial del estudio estuvo compuesta de 285 profesionales de enfermería, cuya media de edad fue de 38.8 años (DT = 10.2) y un rango comprendido entre los 22 y los 63 años. De los profesionales encuestados, 244 eran mujeres y 41 (14.4\%) hombres. $186(65.3 \%)$ de los participantes eran enfermeros, 74 (26.0\%) auxiliares de enfermería, y 25 (8.7\%) tenían cargos de mando. La mayoría de los participantes indicaron ser fijos (38.6\%) o interinos (45.1\%), así como tener un trabajo organizado en turnos rotatorio (69.8\%). La experiencia profesional media de la muestra era de 15.2 años (DT $=10.1$ ), mientras que su antigüedad en la institución y en su puesto de trabajo fueron de 12.6 años $(D T=10.0)$ y 7.3 años $(D T=7.7)$, respectivamente.

\section{Instrumentos}

1. Cuestionario de Datos Sociodemográficos y Laborales elaborado ad hoc para el estudio. Este cuestionario recoge variables sociodemográficas y laborales potencialmente relacionadas con el hostigamiento psicológico. 
2. Cuestionario de Hostigamiento Psicológico en el Trabajo-Revisado (HPT-R) ${ }^{(20)}$. Dicho instrumento está dividido en 2 partes, la primera se compone de 35 ítems y evalúa la frecuencia de conductas de hostigamiento estrictamente de tipo psicológico, donde los encuestados utilizan una escala tipo Likert de 0 a 6 puntos para su contestación. La segunda parte se completa con preguntas dicotómicas cuyo objetivo es identificar la percepción de hostigamiento en los últimos 6 meses previos y/o en el momento del estudio. Además de identificar la procedencia del mobbing, el HPT-R indaga si el encuestado ha sido o no testigo de actos hostiles en su trabajo. El instrumento cuenta con una fiabilidad del 0.96 y sus ítems se agrupa alrededor de 5 factores (Humillación y Rechazo Personal, Desprestigio Profesional, Rechazo Profesional y Violación de la Intimidad, Degradación Profesional y Ninguneo o Aislamiento Profesional) que explicaron el $50.6 \%$ de la varianza.

3. Cuestionario de Síntomas de Estrés ${ }^{(21)}$. Con una fiabilidad de 0.96, éste instrumento consta de 30 posibles síntomas de estrés que la persona pudiera manifestar ante una situación estresante. Se mide su frecuencia e intensidad a través de una escala tipo Likert de intensidad progresiva.

\section{Análisis estadístico}

Los análisis estadísticos fueron realizados mediante el paquete informático Statistical Package for Social Sciences (SPSS) versión 19 para Windows. Se llevaron a cabo análisis descriptivos e inferenciales entre las variables dependientes y su relación con el hostigamiento psicológico en el trabajo y los síntomas de estrés. Los contrastes de medias se realizaron mediante la prueba $t$ de Student. Para las diferencias significativas se tuvo en consideración una probabilidad de 0.50 .

\section{RESULTADOS}

Para responder al objetivo del estudio y obtener una mayor fiabilidad en los resultados, sólo se analizaron los datos reportados por las personas no víctimas, siendo éstas testigos o no testigos de los actos hostiles. En este sentido, la muestra se redujo de 285 a 204 personas, de los cuales 32 son hombres y 172 mujeres.

Conforme a lo planteado en el primer objetivo, los análisis de frecuencias demostraron que el $28.4 \%$ de los participantes $(n=58)$ indicaron haber testificado el hostigamiento de sus compañeros, siendo las enfermeras asistenciales las testigos más habituales de los actos hostiles (75.9\%), seguidas de las auxiliares (19.0\%) y las enfermaras en cargo de mando (5.2\%). La mayoría de testigos fueron mujeres $(89.7 \%)$, estaban casadas (50.0\%) y no tenían hijos (58.6\%) y sólo el $12.1 \%$ de ellos trabajaba también en otra institución. Muchos de los testigos indicaron una baja (44.8\%) o nula $(20.7 \%)$ participación en la toma de decisiones y la inmensa mayoría señalaron que la enfermería es una profesión que está poco valorada (72.0\%). Además, el 36.2\% manifestó el deseo de abandonar su profesión (Tabla I). 


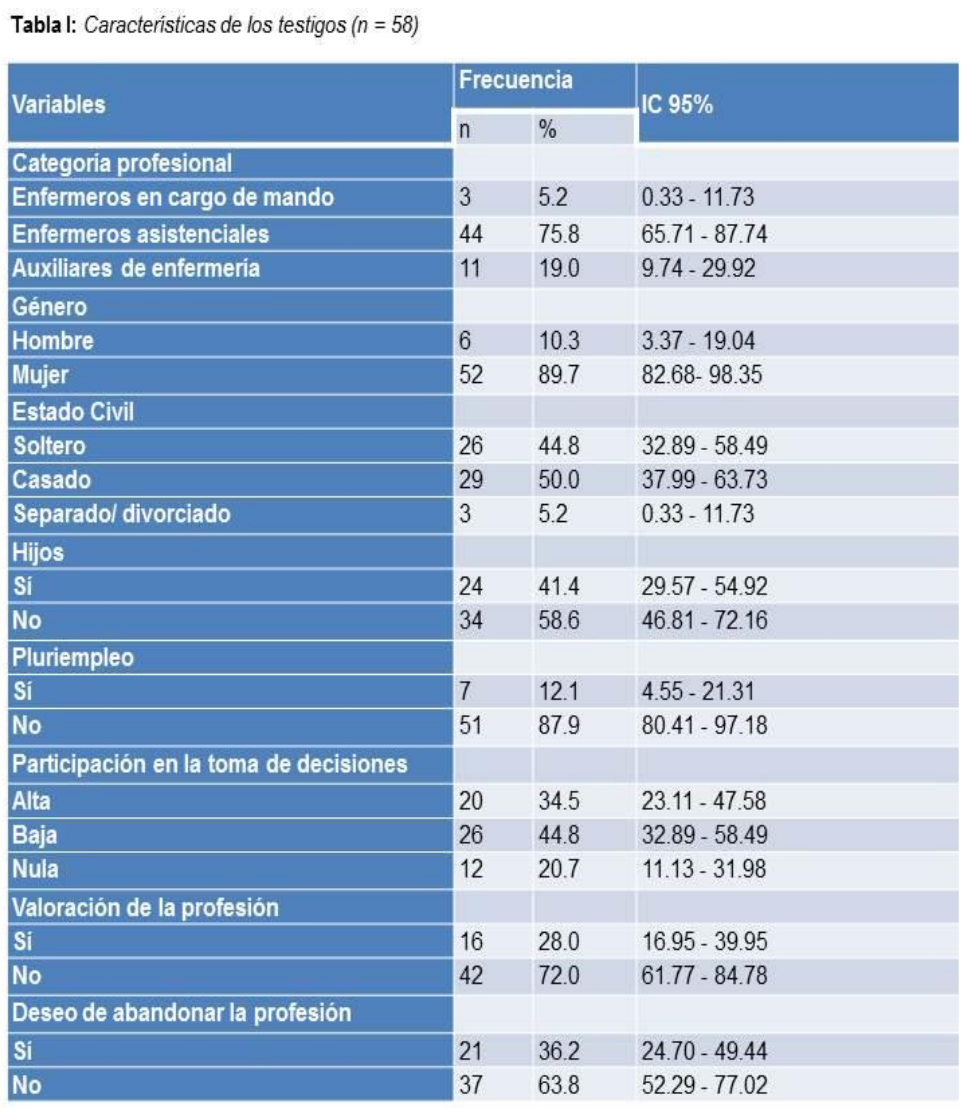

Respecto al segundo objetivo, los resultados indican que la mayoría de los comportamientos hostiles observados por los testigos en su lugar de trabajo estaban relacionados con los problemas de comunicación y relación interpersonal. Se observó que conductas como "Exposiciones negativas o de duda sobre su responsabilidad o capacidad profesional", "Exponerle a críticas por parte del grupo" o "Recibir críticas directas sobre la forma de realizar su trabajo", fueron ampliamente señaladas.

Para una mejor comprensión de los resultados, elegimos agrupar las conductas hostiles observadas según los factores (F) del HPT-R. Así, las conductas hostiles que componen el F1, Humillación y el Rechazo personal, fueron observadas una media de 5.4 veces (DT = 4.0). El F2, Desprestigio Profesional, presentó una media 8.3 (DT = 7.8), el F3, Rechazo Profesional y la Violación de la Intimidad, de $3.6(\mathrm{DT}=2.2)$, el F4, Degradación Profesional, de $2.5(\mathrm{DT}=3.1)$ y el F5, Ninguneo o Aislamiento Profesional, una media de $5.7(\mathrm{DT}=3.0$ ) conductas hostiles observadas (Figura 1).

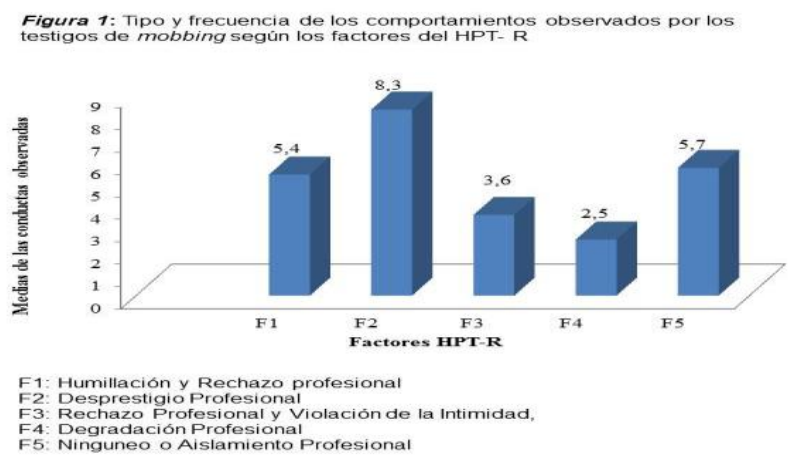


Respecto a lo planteado en el tercer objetivo, los resultados muestran que los testigos padecen significativamente más síntomas de estrés que los no testigos. Así, observamos que los testigos presentaron medias más elevadas para la inmensa mayoría de los síntomas de estrés analizados. Síntomas de estrés como Nervioso (M $=1.67 ; \mathrm{DT}=.96)$, Cansado, falto de energía $(\mathrm{M}=1.36$; $\mathrm{DT}=.81)$, Preocupado $(\mathrm{M}=$ 1.43; DT = .94), Pensado que la gente me mira mal, o piensa mal de mí $(\mathrm{M}=.57$; DT $=.92)$, o Inquieto, sobresaltado $(M=1.12$; DT $=.94)$, fueron los más señalados por los observadores de mobbing (Tabla II).

Tabla II. Comparación entre la percepción de los síntomas de estrés manifestados por testigos y no testigos de hostigamiento psicológico en el trabajo.

\begin{tabular}{|c|c|c|c|c|}
\hline $\mathbf{N}^{0}$ & Sintomas de estrés & Testigos (58) & No testigos (146) & Significación estadistica \\
\hline & & M (DT) & M (DT) & $\mathrm{p}$ \\
\hline 1 & Mareado & $.37(.64)$ & $.30(.54)$ & 475 \\
\hline 2 & Cansado, falto de energia & $1.36(.81)$ & $1.10(.79)$ & .036 \\
\hline 3 & Nervioso & $1.67(.96)$ & $1.31(.87)$ & .011 \\
\hline 4 & Oprimido, con el cuerpo tenso & $1.13(.95)$ & $.95(.92)$ & .234 \\
\hline 5 & Asustado, como con sensación de estar siendo amenazado & $60(.84)$ & $.42(.73)$ & .143 \\
\hline 6 & Con poco apetito & $.64(.87)$ & $.42(.70)$ & .097 \\
\hline 7 & Con taquicardia & $.88(.94)$ & $.59(.82)$ & .034 \\
\hline 8 & Desesperanzado & $96(.86)$ & $.58(.86)$ & .005 \\
\hline 9 & Inquieto, sobresaltado & $1.12(.94)$ & $.73(.86)$ & .006 \\
\hline 10 & Con fallos de memoria & $.76(.82)$ & $.59(.80)$ & .191 \\
\hline 11 & Con dolores en el pecho o dificultades para respirar & $.28(.65)$ & $.23(.52)$ & .579 \\
\hline 12 & Con sentimientos de culpa & $.65(.81)$ & $.44(.73)$ & .084 \\
\hline 13 & Preocupado & $1.43(.94)$ & $1.09(.85)$ & .020 \\
\hline 14 & Con dolores musculares, como reumáticos & $.53(.80)$ & $.74(.95)$ & .143 \\
\hline 15 & Pensado que la gente me mira mal, o piensa mal de mí & $.57(.92)$ & $.28(.56)$ & .028 \\
\hline 16 & Con temblores & $38(.67)$ & $.22(.56)$ & .123 \\
\hline 17 & Con dificultades para pensar con claridad & $.86(.93)$ & $.45(.68)$ & .004 \\
\hline 18 & Fracasado, pensando que no valgo para nada & $.55(.82)$ & $.30(.61)$ & .038 \\
\hline 19 & Tenso & $1.19(.94)$ & $.86(.90)$ & .024 \\
\hline 20 & Inferior a otras personas & $.57(.81)$ & $.37(.72)$ & .115 \\
\hline 21 & Con partes del cuerpo entumecidas, envaradas, adormecidas & $.34(.66)$ & $.24(.59)$ & .251 \\
\hline 22 & Irritable & $1.29(.97)$ & $1.02(.94)$ & .070 \\
\hline 23 & Pensando cosas que no puedo quitarme de la cabeza/mente & $1.19(.93)$ & $.79(.90)$ & .005 \\
\hline 24 & Sin interés por las cosas & $.60(.77)$ & $.36(.70)$ & .035 \\
\hline 25 & Infeliz, deprimido & $.54(.78)$ & $.41(.72)$ & .300 \\
\hline 26 & Con ataques de pánico & $.18(.54)$ & $.13(.43)$ & .530 \\
\hline 27 & Con debilidad en algunas partes del cuerpo & $.40(.67)$ & $.32(.66)$ & .485 \\
\hline 28 & Incapaz de concentrarme & $.91(.89)$ & $.55(.71)$ & .003 \\
\hline 29 & Con insomnio, con sueño intranquilo o con pesadillas & $.84(92)$ & $.70(.91)$ & .327 \\
\hline 30 & Me despierto muy temprano y me cuesta volver a dormir & $.84(1.01)$ & $.66(.88)$ & .194 \\
\hline 31 & Otros & $.54(1.05)$ & $.10(.30)$ & .165 \\
\hline
\end{tabular}

\section{DISCUSIÓN}

En la revisión bibliográfica previa a este trabajo, quedo patente la carencia de estudios que analizan el hostigamiento psicológico en enfermería desde la perspectiva exclusiva de los testigos, por lo que podríamos hablar de un estudio novedoso. Por otra parte, los resultados encontrados también ponen en entredicho la principal característica del mobbing, su subjetividad, no solo porque el porcentaje de testigos es elevado, sino que las conductas hostiles perpetradas en el ámbito laboral pueden llegar a ser manifiestas e, incluso, cuantificables.

Los resultados de este estudio muestran que aproximadamente un tercio del personal de enfermería son testigos del maltrato psicológico que sufre sus compañeros en el trabajo, siendo las mujeres las observadoras habituales del mismo. Este hecho se podría atribuir a que la enfermería es un colectivo predominantemente femenino, o bien a que las enfermeras se muestran más sensibilizadas a la agresión que padecen otras compañeras de trabajo. 
En cuanto a los comportamientos hostiles más observados, los resultados demuestran que el ataque directo a la profesión de enfermería, el abuso verbal y el rechazo profesional fueron las conductas hostiles más frecuentemente observadas. Dichos resultados corroboran estudios previos ${ }^{(4,8,22)}$ al poner de manifiesto que el hostigamiento psicológico en enfermería consiste básicamente en el ataque personal, la erosión de la competencia y reputación profesional y en la obstaculización del trabajo $^{(6,23)}$. La mayoría de estos ataques son perpetrados en público, lo que provoca sentimientos de degradación y debilidad ${ }^{(5,6)}$, también por parte de aquellos que lo observan. En este sentido, las evidencias indican que los testigos se enfrentan a un difícil dilema ante el alcance de los actos observados y su propia impotencia, lo que los induce a una conducta pasiva, de autoprotección ${ }^{(16)}$. Dicha conducta podría no sólo impulsar a justificar los comportamientos observados, sino también a contribuir a una mayor tolerancia a la injusticia y, en definitiva, a la complicidad al maltrato ${ }^{(10)}$. Como señalan algunos autores ${ }^{(18)}$, los testigos tienen miedo de enfrentarse a los agresores, lo que los lleva a abandonar a las víctimas a su propia suerte, de este modo, son pocos los testigos que se levantan en defensa de la víctima. Este sentimiento de impotencia e indefensión podría justificar a que la mayoría de los testigos, en este estudio, indicasen una baja o nula participación en la toma de decisiones que atañe a su trabajo; que la enfermería está poco valorada o, lo más importante, el elevado porcentaje de personas que se plantean abandonar la profesión.

Respecto a la baja participación en la toma de decisiones, ésta podría estar asociada, entre otras cosas, a la condición laboral que este colectivo viene arrastrando a lo largo de su historia ${ }^{(18,24)}$. En este sentido, estudios previos sugieren que la gestión en enfermería se basa más en el miedo que en el respeto ${ }^{(5,23)}$, donde los cambios en su dinámica laboral y profesional no suelen tener en cuenta ni la formación ni la experiencia del profesional ${ }^{(25)}$. Además, el rol técnico de la enfermería suele estar supeditado a la práctica médica, es decir, a un colectivo históricamente más poderoso $^{(23,26)}$, lo que acentúa su tendencia a adoptar comportamientos sumisos y dependientes $^{(4)}$. Todo este contexto, asociado a una formación académica deficitaria en el desarrollo de un pensamiento crítico frente a la hostilidad laboral ${ }^{(23)}$, hace de la enfermería una profesión muy vulnerable a los conflictos cotidianos, que se convierten, en muchos casos, en situaciones de compleja hostilidad.

En cuanto a la percepción de que la enfermería está poco valorada y el elevado deseo de abandonar la profesión manifestado por los testigos, los resultados encontrados corroboran trabajos previos ${ }^{(1,18)}$. De este modo, los testigos se mostraron indirectamente afectados por los comportamientos observados, siendo éstos tolerados e incluso potenciados por la organización ${ }^{(17,23,27)}$. La literatura pone en evidencia que las instituciones de salud otorgan escasa importancia a las denuncias de maltrato psicológico realizadas por parte del personal de enfermería ${ }^{(23,24)}$. Además, son organizaciones que basan su política en la eficiencia productiva y en la contención de costes ${ }^{(6)}$, y no en responder a las preocupaciones manifestadas por su personal $^{(1,23)}$. En este sentido, hay evidencias de que algunas supervisoras de enfermería abogaban por el uso de la intimidación como un método para cumplir las metas establecidas ${ }^{(15,23)}$. Este hecho hace que los testigos se sientan impotentes ante el poder de los hostigadores ${ }^{(17)}$, una vez que éstos no solo no reciben sanciones efectivas por su conducta ${ }^{(5)}$, sino que, pueden incluso ser beneficiados con promociones o tratos de favor $^{(27)}$. Por otra parte, se ha puesto de manifiesto ${ }^{(27)}$ que los testigos perciben un escaso apoyo por parte de los supervisores y un entorno laboral es demasiado permisivo. La percepción de pertenecer a un colectivo vulnerable a 
conductas tan abusivas y la impotencia ante ellas son factores que podrían justificar que un elevado porcentaje de testigos se plantee abandonar su profesión ${ }^{(10)}$, sobre todo los más jóvenes ${ }^{(23)}$.

Nuestros resultados confirman hallazgos previos $^{(1)}$ al poner en evidencia que los testigos de hostigamiento padecen significativamente más síntomas de estrés que los no testigos. Los síntomas de ansiedad, como el nerviosismo, la taquicardia, la inquietud, el sobresalto o la desesperanza, fueron significativamente más señalados por los testigos que por los no testigos. Otros síntomas que también mostraron diferencias significativas entre testigos y no testigos, fueron la dificultad de pensar con claridad o de concentrarse en el trabajo, el sentimiento de fracaso o la pérdida de interés por las cosas. Así, se podría decir que el efecto acumulativo del hostigamiento $^{(6)}$ se extiende más allá de las víctimas, alcanzando también a aquellos que lo observan. De hecho, los síntomas presentados por los testigos son muy similares a los señalados por las víctimas del maltrato psicológico laboral ${ }^{(1,23)}$, lo que repercute negativamente sobre su desempeño ${ }^{(5,8)}$ y, consecuentemente, sobre la atención prestada a los usuarios ${ }^{(18)}$. No obstante, aunque los resultados obtenidos en este trabajo parecen establecer una relación clara entre presenciar el hostigamiento psicológico de otros y percibirse afectados por ello, hay que ser cautos en cuanto a estas interpretaciones, pues no todos los testigos se ven afectados por las conductas observadas pudiendo ser interpretarlas como un comportamiento casual o incluso gracioso $^{(2)}$. En muchos casos, sólo la víctima y los perpetradores conocen la verdadera naturaleza de la hostilidad.

\section{CONCLUSIONES}

El estudio en cuestión podría resultar novedoso, puesto que recoge informaciones exclusivamente de los testigos del hostigamiento psicológico laboral, cuando estos no se percibían víctimas del mismo. Además, la muestra en cuestión pertenece a uno de los colectivos más afectados por ello: la enfermería.

Los resultados obtenidos ponen en entredicho la base en que se construye la definición del hostigamiento psicológico laboral: su subjetividad. El elevado número de testigos encontrados y la información aportada por ellos sugieren que estamos ante a un fenómeno que se basa en conductas socializadas y que son potencializadas por múltiples factores organizacionales. Los comportamientos hostiles testificados por el personal de enfermería corroboran el relato de muchas víctimas, al poner de manifiesto que los problemas de comunicación y relaciones interpersonales, como por ejemplo el abuso verbal o las críticas directas, son una constante en el marco de las conductas hostiles.

Los síntomas de estrés presentados por los testigos nos llevan a pensar que estamos ante una profesión muy vulnerable al maltrato psicológico. Las repercusiones negativas de mobbing se extienden más allá de las víctimas o, en nuestro caso, los testigos, pudiendo alcanzar a los usuarios del sistema sanitario. También se hace notable el elevado porcentaje de testigos que plantean dejar la enfermería.

Los resultados de este estudio podrían contribuir a un mayor conocimiento del fenómeno, a plantear nuevos retos de cómo lidiar con él. Una de las posibles propuestas sería la implantación de formación universitaria donde los alumnos tomasen conocimiento del fenómeno y aprendiesen técnicas efectivas para afrontarlo. 
Otra propuesta iría dirigida a las instituciones colegiales y sindicales, para que concienciasen a los profesionales de la importancia del sentimiento de grupo y de los muchos beneficios que se podrían obtener. La formación continuada y la percepción de apoyo por parte de los compañeros también se podrían trabajar más asiduamente. En definitiva, las posibilidades para poner fin al maltrato laboral podrían ser solo una cuestión de sentido común. Este hecho se hace más relevante una vez que numerosos estudios alertan que la hostilidad contra este colectivo es un problema a escala mundial.

\section{REFERENCIAS}

1. Hansen AM, Hogh A, Persson R, Karlson B, Garde AH, Ørbaek P. Bullying at work, health outcomes, and physiological stress response. J Psychosom Res. 2006; 60(1): 63-72.

2. Einarsen S, Hoel H, Zapf D, Cooper C L. The concept of bullying and harassment at work: The European tradition. En: S. Einarsen, H. Hoel, D. Zapf, \& C. L. Cooper (Eds.), Bullying and Emotion Abuse in harassment in the Workplace (pp. 340). Taylor \& Francis: London, 2011.

3. Lazarus R S, Folkman S. Estrés y procesos cognitivos. Martínez Roca: Barcelona, 1986.

4. Barbosa R, Labronici LM, Sarquis LMM, Mantovani MF. Violência psicológica na prática profissional da enfermeira. Rev Esc Enferm USP. 2011; 45(1): 26-32.

5. Efe SY, Ayaz S. Mobbing against nurses in the workplace in Turkey. Int Nurs Rev. 2010; 57(3): 328-334.

6. Hutchinson $\mathrm{M}$, Vickers $\mathrm{MH}$, Wilkes $\mathrm{L}$, Jackson D. A typology of bullying behaviours: the experiences of Australian nurses. J Clin Nurs. 2010; 19(15-16): 23192328.

7. Hoel H, Sheehan M, Cooper C, Einarsen S. Organisational effects of workplace bullying. En: S. Einarsen, H. Hoel, D. Zapf, \& C. L. Cooper (Eds.), Bullying and Emotion Abuse in harassment in the Workplace (pp. 3-40). Taylor \& Francis: London, 2011.

8. Bambi S, Becattini G, Giusti GD, Mezzetti A, Guazzini A, Lumini E. Lateral hostilities among nurses employed in intensive care units, emergency departments, operating rooms, and emergency medical service. Dimens Crit Care Nurs. 2015; 33(6): 347-354.

9. Hogh $A$, Hoel $H$, Carneiro IG. Bullying and employee turnover among healthcare workers: a three-wave prospective study. J Nurs Manag. 2011; 19(6): 742751.

10. Vessey JA, Demarco RF, Gaffney DA, Budin WC. Bullying of staff registered nurses in the workplace: A preliminary study for developing personal and organizational strategies for the transformation of hostile to healthy workplace environments. J Prof Nurs. 2009; 25(5): 299-306.

11. Sims RL, Sun P. Witnessing workplace bullying and the Chinese manufacturing employee. J Manag Psychol. 2012; 27(1): 9-26.

12. Agervold M. Bullying at work: A discussion of definitions and prevalence, based on an empirical study. Scand J Psychol. 2007; 48(2): 161-172.

13. Berry PA, Gillespie GL, Gates D, Schafer J. Novice Nurse Productivity Following Workplace Bullying. J Nurs Scholarsh. 2012; 44(1): 80-87.

14. Simons SR, Mawn B. Bullying in the workplace - A qualitative study of newly licensed registered nurses. AAOHN. 2010; 58 (7): 305-311.

15. Lewis MA. Nursing bullying: Organizational considerations in the maintenance and perpetration of health care bullying cultures. J Nurs Manag. 2006; 14(1): 52-58. 
16. Lindy C, Schaefer F. Negative workplace behaviours: an ethical dilemma for nurse managers. J Nurs Manag. 2010; 18(3): 285-292.

17. Katrinli A, Atabay G, Gunay G, Cangarli BG. Nurses' perceptions of individual and organizational political reasons for horizontal peer bullying. Nurs Ethics. 2010; 17(5): 614-627.

18. Johnston $M$, Phanhtharath $P$, Jackson BS. The bullying aspect of workplace violence in nursing. Crit Care Nurs Q, 2009; 32(4): 287-295.

19. EScribà V, Más $R$, Cárdenas $M$, Burguete $D$. Fernández R. (). Estresores laborales y bienestar psicológico. Rev Rol Enfer. 2000; 23(7-8): 506-511.

20. Fornés J, Martínez-Abascal MA, García de la Banda G. Análisis factorial del cuestionario de hostigamiento psicológico en el trabajo en profesionales de enfermería. Intern J Clin Health Psychol. 2008; 8(1): 267-283.

21. Manassero MA, Vázquez A, Ferrer VA, Fornés J, Fernández MC. Estrés y burnout en la enseñanza.: Universitat de les Illes Balears (UIB): Palma de Mallorca, (2003).

22. Fornés J, Cardoso M, Castelló JM, Gili M. Psychological harassment in the nursing workplace: An observational study. Arch Psychiatr Nurs. 2011; 25(3): 185-194. 23. Castellón AM. Hostigamiento laboral: amenaza permanente para enfermería. Enferm. Glob. 2012; 11(28): 120-136.

24 Castellón AM. Occupational violence in nursing: Explanations and coping strategies. Rev Lat Am Enferm, 2011; 19(1): 156-163.

25. Pérez-Andrés $C$, Alameda $A$, Albéniz C. La formación práctica en enfermería en la escuela universitaria de enfermería de la Comunidad de Madrid. Opinión de los alumnos y de los profesionales asistenciales. Un estudio cualitativo con grupos de discusión. Rev Esp Salud Públ. 2002; 76(5): 517-530.

26. Johnson S L, Rea R E. Workplace bullying. Concerns for nurse leaders. J Nurs Adm. 2009; 39(2): 84-90.

27. Hutchinson $M$, Wilkes $L$, Jackson D, Vickers $M$. Integrating individual, work group and organizational factors: testing a multidimensional model of bullying in the nursing. J Nurs Manag. 2010; 18(2): 173-181.

28. Domínguez V, Regidor E, de Juanes JR, Calle ME. Sistemas de información sanitaria, registros, encuestas de salud y sistemas de notificación. En: G. Piédrola. (Ed.), Medicina Preventiva y Salud Pública (pp. 51-60). Masson: Barcelona, 2002.

Recibido: 13 de enero 2015; Aceptado: 28 de febrero 2015

ISSN 1695-6141

๑) COPYRIGHT Servicio de Publicaciones - Universidad de Murcia 